\title{
Competency-based medical education: Its time has arrived
}

\author{
Mark F. Levine, MBBCh • George Shorten, MD, PhD
}

Received: 20 January 2016/Revised: 29 January 2016/Accepted: 22 March 2016/Published online: 30 March 2016

(c) Canadian Anesthesiologists' Society 2016

In 2015, the Royal College of Physicians and Surgeons of Canada launched "Competence By Design" (CBD), a multi-year initiative designed to transform residency training in Canada using the principles of competencybased medical education (CBME). Departments of anesthesia in Canadian universities will be implementing a CBD curriculum with the cohort of new residents starting in July 2017. As described in this issue of the Journal, the Department of Anesthesiology at the University of Ottawa enrolled its first cohort of residents into an innovative competency-based program in July 2015. ${ }^{1}$ Many department chairs, clinicians, funding agencies, and other stakeholders may ask why we should change the way we've been training physicians for the past 100 years. They well recognize that we already turn out the most highly educated physicians in history and would be hard pressed to show that most are not competent. ${ }^{2}$

Are we currently educating residents in the most effective and efficient way possible to ensure they are prepared for certification, transition to practice, ongoing maintenance of certification, and development of advanced expertise? All stakeholders, including patients, hospital administrators, and governments, are increasingly demanding greater accountability and assurance that residents are trained in the most cost-effective manner, that specialists graduate with all the abilities required for independent practice, and that they continuously and

M. F. Levine, MBBCh ( $\bowtie)$

Department of Anesthesia and Pain Medicine, The Hospital for Sick Children, The University of Toronto, Toronto, ON, Canada e-mail: mark.levine@utoronto.ca

G. Shorten, $\mathrm{MD}, \mathrm{PhD}$

Department of Anaesthesia and Intensive Care Medicine, University College Cork, Cork, Ireland transparently maintain and improve their performance. In our existing system, many trainees feel inadequately prepared for independent practice on graduation from residency. ${ }^{3}$ This is despite the fact that our training programs are perceived as very high standard and there is a high overall pass rate at the Royal College certification examinations. One of the guiding principles of CBME is to develop a program designed for graduates to achieve certain predefined outcomes -i.e., the competencies to practice safely and independently. The CBME methods and assessment tools are selected or developed to achieve these outcomes. $^{4}$

Most jurisdictions have introduced some form of restricted duty hours, resulting in a significant reduction in time available for residency training, particularly for acquisition of clinical experience. It is therefore imperative that we rethink the way we currently train residents to ensure the training is more focused, effective, efficient, and cost-effective. ${ }^{5}$ Despite the reduction in total training time, the majority of residents still successfully complete residency and achieve specialty certification within the prescribed period. Does this signify that each graduate is ready for safe independent practice or that there are current limitations in measuring performance in a valid and reliable way?

The inability of programs to identify underperforming residents, particularly early in training, may be compounded by the well-recognized concept of the "failure to fail". Dudeck et al. identified lack of documentation and "a lack of knowledge of what to specifically document" as two significant barriers to reporting a failing undergraduate student. ${ }^{6,7}$ Currently, residents are most commonly evaluated using poorly defined non-standardized metrics. In a CBME system, evaluators will need to use formative assessments based on 
identifiable criteria and repeated observations. ${ }^{2,8}$ Residents and educators must be provided with precisely defined criteria for all stages of training to ensure the evaluation process is fair, transparent, and meaningful. Novel evaluation tools will be required to meet the need for many accurate, valid, and reliable assessments that provide formative feedback to trainees. These evaluations should enable educators to identify underperforming trainees earlier in training and provide them with more timely and effective interventions.

In an ideal learner-centred CBD program, it should be feasible for a trainee to respond to the following simple questions for each skill or competence:

1. How good am I at $X$ ?

2. Is that good enough?

3. How exactly do I improve at $X$ ?

Attempting to answer these questions will help identify the various "tools" that are necessary to make CBME succeed in practice, namely, precisely characterized skills, valid and reliable forms of assessment, feedback required to inform deliberate practice, and the environment, equipment, and guidance to carry out the task. Most difficult of all is the need to establish a specific objective standard below which a trainee would not be deemed competent.

The need for these "tools" inevitably prompts consideration of the challenges inherent in implementing such a program in the real world. Fraser et al. thoroughly describe these challenges and explain how the University of Ottawa Department of Anesthesiology proposes to address them. The data generated from their pioneering work could be used to deal with important "derivative" questions.

Such questions to address in the short term (i.e., three to five years) are:

1. What are the characteristics of skills acquisition and attrition during residency (as a function of trainee, skill, practice, and clinical learning opportunity)?

2. Assuming assessment is based on established benchmarks, how does performance vary across a cohort of residents? Is there a large variation in the ability to acquire the requisite skills and the time it takes to do so?

3. Are certain skills prerequisites for or contingent upon others? (If so, it may not be necessary to assess each skill if a consistent hierarchy or dependency structure exists).

4. This ultimately leads to this fourth and fundamentally important question:

Is this mode of training more effective than the best alternative in terms of performance in a simulated setting or clinical outcome? $?^{9,10}$ As there is a paucity of information comparing CBME with traditional training models, introduction of this model should be seen as a significant opportunity for scholarship. ${ }^{5}$

With a greater emphasis on frequent formative feedback, training will have a more learner-centred focus where time becomes a resource rather than a specific endpoint for training.

The new CBD framework has been conceptualized as a hybrid of time (where most residents would be expected to complete training in approximately five years) and competency (where each resident will be able to demonstrate acquisition of the defined competencies at their own pace). ${ }^{11}$ This hybrid model will address some of the concerns related to a pure CBME model, such as planning clinical rotations, service needs, and the acquisition of certain skills that require both time and experience (e.g., anticipation and coordination of attention, which is particularly important in anesthesia). ${ }^{12}$ Within the CBD framework, the phases of training have been redefined to reflect the stages of progress throughout a physician's career. These phases include Transition to Discipline, Foundations of Discipline, Core of Discipline, Transition to Practice and ultimately to Continuing Professional Development and Transition out of Professional Practice. Well-defined criteria for progression at each stage will provide learners and educators with a clear understanding of the requirements for advancement.

A very relevant concern amongst many clinicians and educators is that CBME will lead to a reductionist approach with the breakdown of skills into checklists and tick boxes and that an assessment of a trainee's ability to integrate all components of complex clinical situations may be undervalued. ${ }^{13,14} \mathrm{~A}$ well-designed CBME curriculum will address these concerns. The importance of learning and demonstrating successful acquisition of specific skills is a crucial component of training, particularly in procedurebased specialties, and the importance of timely and effective training (e.g., use of simulation prior to application in clinical practice) and assessment has been well established. ${ }^{13,15}$ Fraser et al. envisage a considerable assessment workload. It is foreseeable that advances in wearable recording devices will soon offer trainees a feasible means of acquiring digital datasets that facilitate selfassessment (with quality control provided by a supervisor) and generate personalized feedback, particularly with respect to specific skills. ${ }^{16}$ Teaching and assessment of integrative clinical abilities have been defined in terms of entrustable professional activities (EPAs) -i.e., the tasks and responsibilities that are essential to the practice of a specialty. ${ }^{17}$ A suite of EPAs is being developed that will encompass the full spectrum of clinical activities relevant to each phase of training. Each EPA will incorporate some, but 
usually not all, Canadian Medical Education Directives for Specialists (CanMEDS) competencies and therefore address some of the concerns relating to the integration of skills into the clinical contexts in which we practice. ${ }^{18}$

Competency-based medical education is the rational and necessary "direction of travel" for training residents. It represents the application of science to optimize human performance in healthcare in a way we take for granted in the development of new drugs or medical devices. In the current environment of greater accountability, the training of doctors will become more effective and transparent. Patients will ultimately benefit. Although there will undoubtedly be challenges and barriers in moving towards a CBME model of training, it is the right thing to do.

\section{L'heure de gloire de la formation médicale fondée sur les compétences est venue}

En 2015, le Collège royal des médecins et chirurgiens du Canada a lancé « La compétence par conception » (CPC), une initiative pluriannuelle dont le but est de transformer la formation des résidents au Canada en se servant des principes de la formation médicale fondée sur les compétences (FMFC). À partir de juillet 2017, les départements d'anesthésie des universités canadiennes mettront en ouvre un programme de CPC pour leurs cohortes de nouveaux résidents. Comme expliqué dans ce numéro du Journal, le département d'anesthésiologie de l'Université d'Ottawa a enrôlé sa première cohorte de résidents dans un programme innovant fondé sur les compétences en juillet 2015 . $^{1}$ Bon nombre de directeurs de département, de cliniciens, d'organismes de financement et d'autres intervenants pourraient s'interroger sur le bien-fondé d'une modification de la formation de nos médecins, largement inchangée depuis un siècle. En effet, ils sont certainement conscients que les médecins que nous formons sont les plus éduqués de l'histoire et auraient bien des difficultés à démontrer que la plupart ne sont pas compétents. $^{2}$

À l'heure actuelle, formons-nous nos résidents de la façon la plus efficace et efficiente qui soit afin de garantir qu'ils sont prêts à être accrédités, à transiter vers la pratique, à continuellement maintenir leur certification et à développer une expertise avancée? Toutes les parties prenantes, qu'il s'agisse des patients, des administrateurs d'hôpitaux ou des gouvernements, s'attendent à une imputabilité toujours accrue et à des garanties toujours plus importantes que les résidents sont formés de la façon la plus rentable possible, que les spécialistes sortent de leur formation munis de toutes les capacités requises pour pratiquer de façon indépendante, et qu'ils entretiennent et fassent avancer leur performance de façon continue et transparente. Dans le système actuel, bon nombre de nos stagiaires ne se sentent pas prêts à pratiquer de façon indépendante lorsqu'ils finissent leur résidence, ${ }^{3}$ et ce malgré le fait que nos programmes de formation soient perçus comme de très haut calibre et qu'il y ait un taux de réussite global élevé des examens de certification du Collège royal. L'un des principes directeurs de la FMFC est de mettre au point un programme conçu de façon à ce que les diplômés atteignent certains objectifs prédéfinis - en d'autres termes, les compétences nécessaires à pratiquer de manière sécuritaire et indépendante. Les méthodes et outils d'évaluation de la FMFC sont sélectionnés et mis au point dans le but d'atteindre ces objectifs. ${ }^{4}$

La plupart des territoires ont introduit des restrictions sur les heures de service sous une forme ou une autre, ce qui entraîne une réduction significative du temps disponible pour la formation des résidents, particulièrement en ce qui touche à l'acquisition de l'expérience clinique. C'est pourquoi il est impératif que nous repensions la façon dont nous formons actuellement nos résidents : leur formation doit être plus concentrée, efficace, efficiente et rentable. ${ }^{5}$ Malgré la réduction du temps total de formation, la majorité des résidents réussissent tout de même leur résidence et obtiennent leur certification de spécialité dans le temps imparti. Doit-on voir dans ce taux de réussite élevé que tous les diplômés sont prêts à pratiquer indépendamment et de façon sécuritaire, ou existe-t-il présentement des écueils dans la façon de mesurer la performance de façon valable et fiable?

L'impuissance des programmes à identifier les résidents qui performent en deçà des exigences, particulièrement au début de la formation, pourrait être encore aggravée par le concept reconnu de "l'absence d'échec » (soit le fait de renoncer à faire échouer quelqu'un). Dudeck et coll. ont déterminé que le manque de documentation et « le manque de connaissances quant à ce qu'il faudrait documenter spécifiquement » constituaient deux obstacles de taille à la 'dénonciation' d'un étudiant en échec. ${ }^{6,7}$ À l'heure actuelle, les résidents sont la plupart du temps évalués à l'aide de mesures à la fois non standardisées et mal définies. Dans un système de FMFC, les évaluateurs devront utiliser des évaluations formatives fondées sur des critères identifiables et des observations répétées. ${ }^{2,8}$ Les résidents et les enseignants doivent disposer de critères précisément définis tout au long des diverses étapes de la formation, ce qui garantira que le processus d'évaluation est équitable, transparent et significatif. Des outils d'évaluation innovants seront nécessaires pour répondre 
au besoin de nombreuses évaluations précises, valables et fiables qui fournissent des rétroactions formatives aux stagiaires. Ces évaluations devraient permettre aux enseignants d'identifier les stagiaires peu performants plus tôt dans leur formation et de leur proposer des interventions plus opportunes et efficaces.

Dans un programme idéal de CPC axée sur l'apprenant, un stagiaire devrait pouvoir répondre aux simples questions suivantes pour chaque compétence ou habileté :

1. Dans quelle mesure suis-je capable de $X$ ?

2. Est-ce suffisant?

3. Comment exactement puis-je améliorer mes compétences pour $X$ ?

En essayant de répondre à ces questions, il sera plus facile d'identifier les divers « outils » nécessaires pour que la FMFC fonctionne en pratique, c'est-à-dire, des compétences précisément décrites, des formes d'évaluation valables et fiables, des rétroactions nécessaires afin d'informer une pratique délibérée, et l'environnement, le matériel et l'orientation pour accomplir la tâche. Le plus difficile est qu'il faut établir une norme objective spécifique en-dessous de laquelle un stagiaire ne sera pas jugé compétent.

Le besoin pour ces « outils » entraîne inéluctablement une réflexion sur les défis inhérents à la mise en œuvre d'un tel programme dans le monde réel. Fraser et coll. décrivent de façon détaillée ces défis et expliquent la façon dont le département d'anesthésiologie de l'Université d'Ottawa se propose de les relever. Les données générées par leurs travaux novateurs pourraient être utilisées pour aborder certaines questions « dérivatives » importantes.

Parmi les questions à aborder à court terme (c.-à-d. trois à cinq ans), citons:

5. Quelles sont les caractéristiques de l'acquisition et de l'attrition des compétences pendant la résidence (en tant que fonction du stagiaire, de la compétence, de la pratique et de l'occasion d'apprentissage clinique)?

6. En prenant pour acquis que l'évaluation se fonde sur des normes établies, dans quelle mesure la performance varie-t-elle dans une cohorte de résidents? Y a-t-il une importante variation dans la capacité à acquérir les compétences requises et le temps nécessaire pour ce faire?

7. Est-ce que certaines compétences préalables doivent être acquises afin de pouvoir en acquérir d'autres ou y en a-t-il certaines qui sont conditionnelles à l'existence d'autres? (Le cas échéant, il pourrait ne pas être indispensable d'évaluer chaque compétence si une hiérarchie ou une structure de dépendance cohérente existe).

8. Ceci nous mène à notre quatrième question, qui est fondamentale:
Ce type de formation est-il plus efficace que la meilleure alternative en termes de performance dans un contexte de simulation ou en matière de résultats cliniques? ${ }^{9,10}$ Étant donné le peu de renseignements comparant la FMFC aux modèles de formation conventionnels, l'introduction de ce modèle devrait être considérée comme une occasion importante d'érudition.

En mettant l'emphase sur des rétroactions formatives plus fréquentes, cette formation se concentrera davantage sur l'apprenant et le temps sera considéré comme une ressource plutôt qu'un critère de formation spécifique.

Le nouveau cadre de CPC a été conceptualisé en tant qu'hybride de temps (la plupart des résidents devraient terminer leur formation en environ cinq ans) et de compétence (chaque résident sera capable de démontrer qu'il a acquis les compétences définies à son propre rythme). ${ }^{11} \mathrm{Ce}$ modèle hybride répondra à certaines des inquiétudes liées à un modèle de FMFC pur, notamment en tenant compte des rotations cliniques, des besoins des services et de l'acquisition de certaines compétences qui nécessitent à la fois temps et expérience (par ex. l'anticipation et la coordination de l'attention, une habileté particulièrement importante en anesthésie). ${ }^{12}$ Dans le cadre de la CPC, les étapes de formation ont été redéfinies afin de refléter les phases d'évolution tout au long de la carrière d'un médecin. Ces phases comprennent le Passage à la discipline, les Connaissances fondamentales de la discipline, les Connaissances approfondies de la discipline, le Passage à la pratique - et, enfin, au Développement professionnel continu et au Retrait de la pratique professionnelle. Des critères clairement définis concernant la réussite de chaque étape et le passage à la suivante permettront aux apprenants et aux enseignants d'avoir une compréhension claire des exigences requises pour progresser.

Une inquiétude tout à fait pertinente parmi bon nombre de cliniciens et d'enseignants est qu'une approche réductionniste découlera de la FMFC car elle réduirait les aptitudes à des listes de contrôle et des cases à cocher et qu'une évaluation de la capacité d'un stagiaire à intégrer toutes les composantes de situations cliniques complexes pourrait être sous-évaluée. ${ }^{13,14}$ Un programme de FMFC bien conçu devra tenir compte de ces inquiétudes. L'importance d'apprendre et de démontrer qu'on a bien acquis des aptitudes spécifiques est une composante essentielle de la formation, particulièrement dans les spécialités qui se fondent sur l'intervention, et le rôle prépondérant d'une formation opportune et efficace (par ex., l'utilisation de la simulation avant l'application en pratique clinique) et l'évaluation sont des faits bien établis. ${ }^{13,15}$ Fraser et coll. envisagent une charge de travail d'évaluation considérable. Il est probable que les 
progrès en matière de dispositifs d'enregistrement portables offriront bientôt aux stagiaires une façon réaliste d'acquérir des ensembles de données numériques qui faciliteront l'auto-évaluation (avec un contrôle de la qualité fourni par un superviseur) et génèreront des rétroactions personnalisées, particulièrement en ce qui touche certaines aptitudes spécifiques. ${ }^{16}$ L'enseignement et l'évaluation d'aptitudes cliniques intégratives ont été définis en termes d'actes professionnels non supervisés (APNS)—c'est-à-dire, les tâches et responsabilités essentielles à la pratique d'une spécialité. ${ }^{17}$ Une série d'APNS est en cours de développement; ces APNS incluront la gamme complète des activités cliniques pertinentes à chaque étape de la formation. Chaque APNS intègrera certaines, mais habituellement pas toutes, compétences CanMEDS, répondant ainsi à certaines des inquiétudes liées à l'intégration des aptitudes dans les contextes cliniques dans lesquels nous pratiquons. ${ }^{18}$

La formation médicale fondée sur les compétences est la « route à suivre », d'un point de vue rationnel autant que nécessaire, pour former nos résidents. Elle représente l'application de la science pour maximiser les performances humaines en soins de santé d'une façon dont nous n'imaginons même pas nous passer dans la mise au point de nouveaux médicaments ou dispositifs médicaux. Dans l'environnement actuel d'imputabilité accrue, la formation de médecins deviendra plus efficace et transparente. En bout de ligne, les patients en seront les gagnants. Bien que la route vers un modèle de FMFC sera certainement parsemée d'embuches et de défis, c'est la meilleure chose à faire.

Conflicts of interest None declared.

Editorial responsibility This submission was handled by Dr. Philip M. Jones, Associate Editor, Canadian Journal of Anesthesia.

Conflit d'intérêt Aucun.

Responsabilité éditoriale Cet article a été traité par Dr. Philip M. Jones, Rédacteur adjoint, Journal canadien d'anesthésie.

\section{References}

1. Fraser AB, Stodel AJ, Chaput AJ. Curriculum reform for residency training: competence, change, and opportunities for leadership. Can J Anesth 2016; 63. DOI: 10.1007/s12630-0160637-7.

2. Iobst WI, Sherbino J, Ten Cate OT, et al. Competency-based medical education in postgraduate medical education. Med Teach 2010; 32: 651-6.

3. Postgraduate Medical Education; University of Toronto. PGME Annual Reports. Annual Report July 12014 - June 30 2015; Page 18. Available from URL: http://pgme.utoronto.ca/content/pgmeannual-reports (accessed January 2016).

4. Frank JR, Snell LS, Ten Cate OT, et al. Competency-based medical education: theory to practice. Med Teach 2010; 32: 63845.

5. Sonnadora RR, Mui C, McQueen S, et al. Reflections on competency-based education and training for surgical residents. J Surg Educ 2014; 71: 151-8.

6. Dudek NL, Marks MB, Regehr G. Failure to fail: the perspectives of clinical supervisors. Acad Med 2005; 80: S84-7.

7. Guerrasio J, Furfari KA, Rosenthal LD, Nogar CL, Wray KW, Aagaard EM. Failure to fail: the institutional perspective. Med Teach 2014; 36: 799-803.

8. Dath $D$, Iobst $W$. The importance of faculty development in the transition to competency-based medical education. Med Teach 2010; 32: 683-6.

9. Birkmeyer JD, Finks JF, O'Reilly A, et al. Surgical skill and complication rates after bariatric surgery. N Engl J Med 2013; 369: 1434-42.

10. Angelo RL, Ryu RK, Pedowitz RA, et al. A proficiency-based progression training curriculum coupled with a model simulator results in the acquisition of a superior arthroscopic Bankart skill set. Arthroscopy 2015; 31: 1854-71.

11. Royal College of Physicians and Surgeons of Canada. Competence By Design (CBD): Speaking Points. Available from URL: http://www.royalcollege.ca/portal/page/portal/rc/ resources/cbme/resources/infosheets_reports_graphics (accessed January 2016).

12. Talbot $M$. Monkey see, monkey do: a critique of the competency model in graduate medical education. Med Educ 2004; 38: 58792.

13. Hawkins RE, Welcher CM, Holmboe ES, et al. Implementation of competency-based medical education: are we addressing the concerns and challenges? Med Educ 2015; 49: 1086-102.

14. Glass JM. Competency based training is a framework for incompetence. BMJ 2014; 348: g2909.

15. Shorten GD, Gallagher AG, Satava RM. The medical procedure pathway: creating a global standard methodology to benefit patients. Eur J Anaesthesiol 2015; 32: 79-82.

16. Paro JA, Nazareli R, Gurjala A, Berger A, Lee GK. Video-based self-review: comparing Google Glass and GoPro technologies. Ann Plast Surg 2015; 74: S71-4.

17. Jonker $G$, Hoff $R G$, Ten Cate $O$. A case for competency-based anaesthesiology training with entrustable professional activities: an agenda for development and research. Eur J Anaesthesiol 2015; 32: 71-6.

18. Royal College of Physicians and Surgeons of Canada. About CanMEDS. Available from URL: http://www.royalcollege.ca/ portal/page/portal/rc/canmeds/about (accessed January 2016). 